\title{
Cognitive Radio with Partial Channel State Information at the Transmitter
}

\author{
Pin-Hsun Lin, Shih-Chun Lin, Chung-Pi Lee, and Hsuan-Jung Su
}

Pin-Hsun Lin, Chung-Pi Lee, and Hsuan-Jung Su are with the Department of Electrical Engineering and Graduate Institute of Communication Engineering, National Taiwan University, Taipei, Taiwan. Shih-Chun Lin is with the Institute of Communications Engineering, National Tsing Hua University, Taipei, Taiwan. Email: \{f89921145, r94942026\}@ntu.edu.tw, hjsu@cc.ntu.edu.tw, and sclin2@ntu.edu.tw. This work was presented in part at the IEEE International Conference on Communications, May 2008, and at the Annual Conference on Information Sciences and Systems, Mar. 2008 and 2009. 


\title{
Cognitive Radio with Partial Channel State Information at the Transmitter
}

\begin{abstract}
In this paper, we present the cognitive radio system design with partial channel state information known at the transmitter (CSIT). We replace the dirty paper coding (DPC) used in the cognitive radio with full CSIT by the linear assignment Gel'fand-Pinsker coding (LA-GPC), which can utilize the limited knowledge of the channel more efficiently. Based on the achievable rate derived from the LA-GPC, two optimization problems under the fast and slow fading channels are formulated. We derive semianalytical solutions to find the relaying ratios and precoding coefficients. The critical observation is that the complex rate functions in these problems are closely related to ratios of quadratic form. Simulation results show that the proposed semi-analytical solutions perform close to the optimal solutions found by brute-force search, and outperform the systems based on naive DPC. Asymptotic analysis also shows that these solutions converge to the optimal ones solved with full CSIT when the $K$-factor of Rician channel approaches infinity. Moreover, a new coding scheme is proposed to implement the LA-GPC in practice. Simulation results show that the proposed practical coding scheme can efficiently reach the theoretical rate performance.
\end{abstract}

\section{INTRODUCTION}

As the demand for high data rate steadily increases, efficient spectrum usage becomes a critical issue. Recent measurements from the Federal Communications Commission (FCC) have indicated that 90 percent of the time, many licensed frequency bands remain unused. A radio technology that promises to solve such problems is the cognitive radio (CR) [1]. This technology is capable of dynamically sensing and locating unused spectrum segments in a target spectrum pool, and communicating via the unused spectrum segments without causing harmful interference to the primary users. The primary users are defined as those of existing commercial standards. If a primary user demands the channel, the CR user should vacate and find an alternative one. Assuming that there is perfect channel state information at the transmitter (CSIT), Devroye et. al. in [2] proposed the concept of interference mitigation $\mathrm{CR}$ in which the $\mathrm{CR}$ and primary users can simultaneously transmit at the same time and frequency bands. The CR transmitter not only transmits its own signal but also relays the primary users'. This is the key to keeping the primary users' transmission rates unchanged under the coexistence of CR users since the CR users' signals may interfere with the primary users'. For the CR receiver, signals from the primary transmitters and those relayed by the CR transmitter are interferences. These interferences are non-causally known at the CR transmitter and can be precoded with dirty paper coding (DPC) [3] such that the CR receiver can decode as if these interferences do not exist. The validity of 
the non-causally known interferences has been discussed in [4]. DPC is a promising precoding technique for cancelling arbitrary interferences perfectly known only at the transmitter but not at the receiver. Devroye et. al. [2] then derived the achievable rate of the DPC based interference mitigation CR system. In [4], Jovicic and Viswanath further proved that the DPC based CR system indeed achieves the capacity.

Due to limited feedback bandwidth and the delay caused by channel estimation, it is more practical to consider the case with only partial CSIT. Unfortunately, the interference-free rate achieved by DPC heavily relies on perfectly known CSIT. It has been observed that naively applying DPC in fading channels with partial CSIT may cause a significant performance loss [5]. In this paper, we focus on the CR system design for the cases where only the fading channel statistics are available at both the primary and CR transmitters. The main contributions of this paper are as following. We use a coding scheme more general than the DPC, termed the linear-assignment Gel'fand-Pinsker coding (LA-GPC) [6] to derive the achievable rate of the CR channel. Based on the achievable rate, we then propose design methods to optimize the performance of the CR system with only the statistics of CSIT for both fast and slow fading scenarios [7]. The goal is to keep the primary user's rate unchanged while maximizing the CR user's rate. The considered problems are non-convex and are almost analytically intractable. Therefore, we provide semi-analytical solutions to the optimal relaying ratio of the CR transmitter in fast and slow fading channels, respectively. These solutions are obtained by expressing the primary user's rate constraint in the ratio of quadratic form, then applying the techniques of moments of quadratic forms and the deterministic approximations of probability inequalities [8]. To optimize the CR user's own rate, we also provide semi-analytical solutions to the precoding coefficients in fast and slow fading channels, respectively. In slow fading channels, we show that the complex objective function can be recast as a compound function of the incomplete gamma function from which the precoding coefficient can be easily numerically solved. Asymptotic analysis is also given to show that these solutions converge to the optimal ones solved with full CSIT when the $K$-factor of Rician channel approaches infinity. This result verifies the correctness of the proposed methods. Simulation results show that the proposed semi-analytical methods perform well compared to the optimal solutions found by brute-force search under various channel conditions. In addition to the theoretical results based on unstructured random Gaussian codebooks, we also use nested-lattice codebooks with lattice decoding to implement the precoding in practice [9]. Simulation results show that the nested-lattice coding scheme can efficiently approach the theoretical achievable rate.

This paper is organized as follows. Section $\coprod$ gives the system model of the CR system with partial CSIT. Review of the LA-GPC and its application to the partial CSIT CR system are also given in this section. The proposed parameter design for fast and slow fading channels 
are discussed in Section $\amalg I-\mathrm{A}$ and Section $\amalg$ III-B, respectively. The asymptotic analysis of the parameters is given in Section IV. A nested-lattice based LA-GPC scheme using the proposed design parameters is illustrated in Section V. Simulation results are given in Section VI. Finally, Section VII concludes this paper.

\section{BACKGROUND AND SYSTEM MODEL}

In this section we first introduce the system model in consideration. We then review the problems of DPC and LA-GPC in fading channels. After that we will derive the rate formula of the fading cognitive channel with LA-GPC.*

\section{A. The fading cognitive channel}

The cognitive channel coined by [2] is shown in Fig. 11. The channel gains between primary transmitter and receiver, primary transmitter and $\mathrm{CR}$ receiver, $\mathrm{CR}$ transmitter and primary receiver, and CR transmitter and receiver are denoted by $H_{11}, H_{21}, H_{12}$, and $H_{22}$, respectively. The primary and CR transmitted signals are $X_{p}$ and $X_{c}$ with transmit power constraint $P_{p}$ and $P_{c}$, respectively. The unidirectional arrow from the primary transmitter to the $\mathrm{CR}$ transmitter means that the CR transmitter unilaterally knows the primary user's codewords non-causally. The feasibility of this assumption is discussed in [4]. The signals at the primary and CR receivers are

$$
\left[\begin{array}{l}
Y_{p} \\
Y_{s}
\end{array}\right]=\left[\begin{array}{ll}
H_{11} & H_{12} \\
H_{21} & H_{22}
\end{array}\right]\left[\begin{array}{cc}
1 & 0 \\
\sqrt{\frac{\alpha_{1} P_{c}}{P_{p}}} & 1
\end{array}\right]\left[\begin{array}{c}
X_{p} \\
\hat{X}_{c}
\end{array}\right]+\left[\begin{array}{c}
Z_{p} \\
Z_{S}
\end{array}\right],
$$

where $\hat{X}_{c}$ is the CR user's own signal after being precoded. The CR transmitter relays the primary user's signal to maintain the performance of the primary link. The relaying ratio $\alpha_{1}$ is the percentage of CR's transmit power used for relaying the primary signal. Thus the factor $\sqrt{\alpha_{1} P_{c} / P_{p}}$ is to ensure that the relaying power is $\alpha_{1} P_{c}$. The circularly symmetric complex additive white Gaussian noises (AWGN) at the primary and CR receivers are denoted by $Z_{p} \sim$ $\mathcal{C N}\left(0, \sigma_{Z_{p}}^{2}\right)$ and $Z_{S} \sim \mathcal{C N}\left(0, \sigma_{Z_{s}}^{2}\right)$, respectively.

In the following discussion we assume that the primary receiver knows $H_{11}$ perfectly, but only the statistics of $H_{11}$ are known at primary transmitter. Similarly, the CR receiver knows all four channel gains perfectly, but only the statistics of them are known at the CR transmitter. We

\footnotetext{
${ }^{*}$ In this paper, the superscript $(.)^{\mathrm{T}}$ and $(.)^{\mathrm{H}}$ denote the transpose and complex conjugate, respectively. Letters without and with underlines denote variables and vectors, respectively. If a letter is Italic capital, the corresponding variable or vector is random. Boldface capital letters denote deterministic matrices. $\mathbf{A}(i, j)$ denotes the entry at the $i$ th row and the $j$ th column of the matrix A. The covariance matrix of the random vector $[A B]^{\mathrm{T}}$ is denoted by $\Sigma_{A, B} .|\mathbf{A}|$ and $|a|$ represent the determinant of the square matrix $\mathbf{A}$ and the absolute value of the scalar variable $a$, respectively. $\mathbf{I}_{n}$ is the identity matrix with dimension $n . I(A ; B)$ denotes the mutual information between $A$ and $B$. The trace of $\mathbf{A}$ is denoted by $\operatorname{tr}(\mathbf{A})$. The diagonal matrix whose diagonal entries are $\underline{a}$ is denoted by $\operatorname{diag}(\underline{a})$. All the logarithm operations are of base 2 such that the unit of rates is in bit.
} 
assume that the primary and CR users are subject to slow or fast fading channels simultaneously. In this paper the four channels $H_{i j}, 1 \leq i, j \leq 2$, are assumed to be Rice distributed with mean and variance denoted by $\mu_{i j}$ and $\sigma_{i j}^{2}$, respectively. We also let $\left|\mu_{i j}\right|^{2}+\sigma_{i j}^{2}=1$ to keep the calculation of the received signal-to-noise ratio (SNR) simple.

\section{B. LA-GPC in fading channels}

Since only the statistics of the fading channels are known at the transmitter, the CR user cannot use the classical DPC to perfectly cancel the interference due to $X_{p}$. Instead, the DPC is replaced by the LA-GPC which is more suitable for this task. For brevity, the CR user's channel is recast as

$$
Y=H_{x} X+H_{S} S+Z
$$

with

$$
H_{s}=H_{21}+\sqrt{\frac{\alpha_{1} P_{c}}{P_{p}}} H_{22}, H_{x}=H_{22}, Y=Y_{s}, X=\hat{X}_{c}, S=X_{p}, \text { and } Z=Z_{s},
$$

where $X, S$, and $Z$ are assumed to be independent complex Gaussian random variables with zero mean and variances (powers) $P_{x}, P_{s}$, and $P_{z}=\sigma_{Z_{s}}^{2}$, respectively. The fading channel gains $H_{x}$ and $H_{s}$ of the signal and interference, respectively, are known perfectly at the receiver, but only their statistics are known at transmitter. For illustration purpose, we first focus on the fast fading channel in this subsection. The capacity of this channel can be modified from [6] as

$$
\sup _{f(u \mid s), f(\cdot)} E\left\{R\left(H_{x}, H_{S}\right)\right\},
$$

where

$$
R\left(H_{x}, H_{S}\right)=I\left(U ; Y \mid H_{x}, H_{S}\right)-I(U ; S)
$$

$U$ is an auxiliary random variable with distribution obtained via the conditional distribution $f(u \mid s)$, and $f(\cdot)$ is a deterministic function such that $X=f(U, S)$. We select $f(U, S)=U-\alpha_{2} S$ which makes,

$$
U=X+\alpha_{2} S .
$$

Such strategy function selection is called the linear assignment strategy and $\alpha_{2}$ is the precoding coefficient. With perfect CSIT, we can choose $\alpha_{2}$ as the linear minimum mean square error (MMSE) filter coefficient $\alpha_{c} \triangleq\left|H_{x}\right|^{2} P_{x} /\left(\left|H_{x}\right|^{2} P_{x}+P_{z}\right)$ to estimate $X$ from the interference-free channel $Y=H_{x} X+Z$, and the interference-free rate is achievable. Costa named the LA-GPC with $\alpha_{2}=\alpha_{c}$ as DPC [3]. However, in our setting, the selection of the filter $\alpha_{2}$ must rely only on the channel statistics. Thus the MMSE filter used in Costa's DPC cannot be used here. 


\section{Achievable rate of $C R$ with $L A-G P C$}

Before further discussing the primary and CR users' performances in fast and slow fading channels, the rate functions with given channel gains should be derived first. Assuming that the linear-assignment strategy is used, we have $U=\hat{X}_{c}+\alpha_{2} X_{p}$ from (3) and (6). The mutual information with given channel realizations can be computed as [10]

$$
\begin{aligned}
R\left(h_{22}, h_{21}\right) & \triangleq I\left(U ; Y_{S} \mid H_{22}=h_{22}, H_{21}=h_{21}\right)-I(U ; S) \\
& =\log \left(\left(\left|h_{22}\right|^{2} \sigma_{\hat{x}_{c}}^{2}+\left|h_{21}+\sqrt{\frac{\alpha_{1} P_{c}}{P_{p}}} h_{22}\right|^{2} P_{p}+\sigma_{Z_{s}}^{2}\right) \sigma_{\hat{x}_{c}}^{2}\right)-\log \left(\left|\Sigma_{U, Y_{s}}\right|\right),
\end{aligned}
$$

where $\sigma_{\hat{x}_{c}}^{2}=\left(1-\alpha_{1}\right) P_{c}$, and $\Sigma_{U, Y_{s}}$ is

$$
\Sigma_{U, Y_{s}}=\left(\begin{array}{cc}
\sigma_{\hat{x}_{c}}^{2}+\left|\alpha_{2}\right|^{2} P_{p} & h_{22}^{*} \sigma_{\hat{x}_{c}}^{2}+\alpha_{2}\left(h_{21}^{*}+h_{22}^{*} \sqrt{\frac{\alpha_{1} P_{c}}{P_{p}}}\right) P_{p} \\
h_{22} \sigma_{\hat{x}_{c}}^{2}+\alpha_{2}^{*}\left(h_{21}+h_{22} \sqrt{\frac{\alpha_{1} P_{c}}{P_{p}}}\right) P_{p} & \left|h_{22}\right|^{2} \sigma_{\hat{x}_{c}}^{2}+\left|h_{21}+h_{22} \sqrt{\frac{\alpha_{1} P_{c}}{P_{p}}}\right|^{2} P_{p}+\sigma_{Z_{s}}^{2}
\end{array}\right) .
$$

Similarly, when the CR user is active, the primary user's rate with given channel realizations can be computed as

$$
\begin{aligned}
R\left(h_{11}, h_{12}\right) & \triangleq I\left(X_{p} ; Y_{p}, H_{11}=h_{11}, H_{12}=h_{12}\right) \\
& =\log \frac{\left(1-\alpha_{1}\right) P_{c}\left|h_{12}\right|^{2}+\left|\sqrt{P_{p}} h_{11}+\sqrt{\alpha_{1} P_{c}} h_{12}\right|^{2}+\sigma_{Z_{p}}^{2}}{\left(1-\alpha_{1}\right) P_{c}\left|h_{12}\right|^{2}+\sigma_{Z_{p}}^{2}} .
\end{aligned}
$$

\section{PARAmeter DESign FOR FADING ChANNEls}

In this section we provide semi-analytical methods for finding the relaying ratios $\alpha_{1}$ and precoding coefficients $\alpha_{2}$ under fast and slow fading channels.

\section{A. Fast fading scenario}

With full CSIT, the CR transmitter must ensure the primary user's rate unchanged as in Viswanath's setting [4]. For fast fading channels, a meaningful definition of such rate is the ergodic capacity [7]. Therefore, we take the rate functions (7) and (9) averaged over all channel realizations to form the following design criteria

$$
\begin{aligned}
& \operatorname{maximize} E\left[R\left(H_{22}, H_{21}\right)\right] \\
& \text { subject to } E\left[R\left(H_{11}, H_{12}\right)\right] \geq E\left[\log \left(1+\frac{\left|H_{11}\right|^{2} P_{p}}{\sigma_{Z_{p}}^{2}}\right)\right] .
\end{aligned}
$$

Since (11) only depends on $\alpha_{1}$ but not $\alpha_{2}$, we can solve (11) for $\alpha_{1}$ and then (10) for $\alpha_{2}$ independently. The key to solving (11) is to transform (9) into a ratio of quadratic form. By resorting to the moments of the quadratic form [11] we can find $\alpha_{1}$ by solving a simple 
logarithmic equation numerically. Thus we first rearrange the terms in (9) into the following matrix form. Let

$$
\underline{H}_{p} \triangleq\left(H_{11}, H_{12}\right)^{\mathrm{T}} \sim \mathcal{C N}(\mu, \Sigma),
$$

$\mu \triangleq\left(\mu_{11}, \mu_{12}\right)^{\mathrm{T}}$, and $\Sigma \triangleq \operatorname{diag}\left(\left[\sigma_{11}^{2} \sigma_{12}^{2}\right]\right)$. Also let

$$
\mathbf{Q} \triangleq\left(\begin{array}{cc}
0 & 0 \\
0 & \left(1-\alpha_{1}\right) P_{c}
\end{array}\right) \text { and } \mathbf{P} \triangleq\left(\begin{array}{cc}
P_{p} & \sqrt{\alpha_{1} P_{c} P_{p}} \\
\sqrt{\alpha_{1} P_{c} P_{p}} & \alpha_{1} P_{c}
\end{array}\right) .
$$

Without loss of generality, we let both $\sigma_{Z_{p}}^{2}$ and $\sigma_{Z_{s}}^{2}$ be 1 . Then the primary user's rate formula becomes

$$
R\left(H_{11}, H_{12}\right)=\log \left(1+\frac{\underline{H}_{p}^{\mathrm{H}} \mathbf{P} \underline{H}_{p}}{\underline{H}_{p}^{\mathrm{H}} \mathbf{Q} \underline{H}_{p}+1}\right) .
$$

Note that the right hand side of (11) is assumed to be a known constant $R_{\text {ergodic }}^{P}$ for the CR transmitter. Similarly, after some manipulations the CR user's rate becomes

$$
R\left(H_{22}, H_{21}\right)=\log \left(\frac{\underline{H}_{c}^{\mathrm{H}}(\mathbf{P}+\mathbf{Q}) \underline{H}_{c}+1}{\underline{H}_{c}^{\mathrm{H}}\left(c_{0}(\mathbf{P}+\mathbf{Q})-\mathbf{D}\right) \underline{H}_{c}+c}\right)+\log \sigma_{\hat{x}_{c}}^{2},
$$

where $\underline{H}_{c}=\left[H_{22}, H_{21}\right]^{T}, c_{0}=\sigma_{\hat{x}_{c}}^{2}+\left|\alpha_{2}\right|^{2} P_{p}, c=c_{0}$, and

$$
\mathbf{D}=\left(\begin{array}{cc}
\left|\alpha_{2}\right|^{2} P_{p}^{2} & \alpha_{2}\left(\sigma_{\hat{\hat{x}}_{c}}^{2}+\alpha_{2}^{*} \sqrt{\alpha_{1} P_{c} P_{p}}\right) P_{p} \\
\alpha_{2}^{*}\left(\sigma_{\hat{x}_{c}}^{2}+\alpha_{2} \sqrt{\alpha_{1} P_{c} P_{p}}\right) P_{p} & \left|\sigma_{\hat{x}_{c}}^{2}+\alpha_{2}^{*} \sqrt{\alpha_{1} P_{c} P_{p}}\right|^{2}
\end{array}\right) .
$$

The optimization problem described in (10) and (11) is not convex. Thus we propose methods described in Theorem 1 and Proposition 1 to solve it sub-optimally with proofs given in Appendix $\mathrm{A}$ and B, respectively.

Theorem 1: The relaying ratio $\alpha_{1}$ for the fast fading CR channel can be found sub-optimally by solving

$$
\log \left(\frac{1+\mu_{\varepsilon_{1}}}{1+\mu_{\varepsilon_{2}}}\right)-\frac{\log e}{2} \cdot \frac{\sigma_{\varepsilon_{1}}^{2}}{\left(1+\mu_{\varepsilon_{1}}\right)^{2}}=R_{\text {ergodic }}^{P}
$$

where

$$
\mu_{\varepsilon_{1}}=\mu^{H} \mathbf{S} \mu+\operatorname{tr}(\Sigma \mathbf{S}), \sigma_{\varepsilon_{1}}^{2}=2 \mu^{H} \mathbf{S} \Sigma \mathbf{S} \mu+\operatorname{tr}(\Sigma \mathbf{S})^{2}, \mu_{\varepsilon_{2}}=\mu^{H} \mathbf{Q} \mu+\operatorname{tr}(\Sigma \mathbf{Q}),
$$

$\mathbf{S}=\mathbf{P}+\mathbf{Q}, \mu$ and $\Sigma$ are defined right after (12), and $\mathbf{P}$ and $\mathbf{Q}$ are defined in (13), respectively.

After knowing $\alpha_{1}$, we can treat (10) as an unconstrained optimization problem. Let $B\left(\alpha_{2}\right) \triangleq$ $\underline{H}_{c}^{\mathrm{H}}\left(c_{0}(\mathbf{P}+\mathbf{Q})-\mathbf{D}\right) \underline{H}_{c}+c$, which is a convex function of $\alpha_{2}$. With the fact that the logarithm is concave, $R\left(H_{22}, H_{21}\right)$ is concave with respect to $\alpha_{2}$. Therefore, from [?, p.209] we know that 
(10) is concave. Thus, we can set the derivative of $E\left[R\left(H_{22}, H_{21}\right)\right]$ with respect to the complex conjugate of $\alpha_{2}$ to zero to find the extreme value

$$
\begin{aligned}
\frac{\partial E\left[R\left(H_{21}, H_{22}\right)\right]}{\partial \alpha_{2}^{*}} & =-\frac{\partial}{\partial \alpha_{2}^{*}} \int \log B\left(\alpha_{2}\right) f_{\underline{H}_{c}} d \underline{H}_{c}=-\int \frac{\partial}{\partial \alpha_{2}^{*}} \log B\left(\alpha_{2}\right) f_{\underline{H}_{c}} d \underline{H}_{c}=-\int \frac{B^{\prime}\left(\alpha_{2}\right)}{B\left(\alpha_{2}\right)} f_{\underline{H}_{c}} d \underline{H}_{c} \\
& =-E\left[\frac{B^{\prime}\left(\alpha_{2}\right)}{B\left(\alpha_{2}\right)}\right]=0
\end{aligned}
$$

where $f_{\underline{H}_{c}}$ is the probability density function of $\underline{H}_{c}$, and the validity of the interchange of the integration and differentiation of the second equality is obvious. Note that solving the general moment of quadratic form [11] in (17) is intractable. Thus we resort to finding the suboptimal solution.

Proposition 1: The precoding coefficient $\alpha_{2}$ for the fast fading CR channel can be approximated by

$$
\alpha_{2}=\frac{\left(\mu_{22}^{*} \mu_{21}+\sqrt{\frac{\alpha_{1} P_{c}}{P_{p}}}\right)\left(1-\alpha_{1}\right) P_{c}}{\left(1-\alpha_{1}\right) P_{c}+1}
$$

\section{B. Slow fading scenario}

For the quasi-static slow fading channels, the decoding error probability cannot be arbitrarily small since the transmitter does not know the reliable transmission rate with the limited channel knowledge. In this channel, the outage probability [7] for a certain target rate is more suitable than the Shannon capacity to measure the performance.

For the CR user with a target rate $R^{C R}$, the outage probability which is defined as $P\left(R\left(H_{21}, H_{22}\right)<\right.$ $R^{C R}$ ) should be minimized. Let $P_{\text {out }}^{P}$ and $R^{P}$ be the primary user's achievable outage probability and outage capacity, respectively, in the absence of the CR transmitter. Since the CR system must ensure that the primary user's outage probability does not increase, we have the following constrained minimization problem for the outage probability of the $\mathrm{CR}$ user

$$
\begin{aligned}
\operatorname{minimize} & P_{\text {out }}^{C R} \\
\text { subject to } & P\left(R\left(H_{21}, H_{22}\right)<R^{C R}\right) \leq P_{\text {out }}^{C R} \\
& P\left(R\left(H_{11}, H_{21}\right)<R^{P}\right) \leq P_{\text {out }}^{P} .
\end{aligned}
$$

Assume that the CR transmitter knows the primary user's target outage probability $P_{\text {out }}^{p}$ and target rate $R^{P}$. Similar to the ergodic case, $\alpha_{1}$ and $\alpha_{2}$ can be solved from (20) and (19) sequentially. To reformulate the complex outage constraint (20) into an analytically solvable one, we resort to the concept of deterministic approximations of probability inequalities [8]. In [10] it was observed that if the mean of the rate is maximized, the deviation of the rate will also be increased, which may result in a worse outage capacity. As a result, if we want to achieve the outage probability constraint efficiently, we need to consider both the mean and deviation of the rate simultaneously. 
In addition, to take the tightness of the approximation into consideration, we adopt the modified Cantelli's inequality [12]. The results are summarized as the following with proofs in Appendix C] and D, respectively. The parameters $\mu_{\varepsilon_{2}}, \Sigma, \mathbf{P}$, and $\mathbf{Q}$ are defined in Theorem 1. With

$$
\triangle_{1} \triangleq \frac{\underline{H}_{p}^{H} \mathbf{Q} \underline{H}_{p}+1}{\underline{H}_{p}^{H} \mathbf{P} \underline{H}_{p}}
$$

we have

Theorem 2: The relaying ratio $\alpha_{1}$ for the slow fading CR channel can be found by solving the following equation

$$
\mu_{\triangle_{1}}+\sqrt{\frac{r}{P_{\text {out }}^{P}}-1} \sigma_{\triangle_{1}}=\frac{1}{2^{R^{P}}-1}
$$

where $\mu_{\triangle_{1}}$ and $\sigma_{\triangle_{1}}$ are the mean and the standard deviation of $\triangle_{1}$, respectively. The constant $r$ can be 1 or $2 / 9$ depending on the $K$-factor.

Since (21) is a ratio of quadratic form, we can resort to [13] to efficiently compute the mean and the variance of $\triangle_{1}$ by the following proposition. Then (22) can be easily solved by a polynomial equation of $\alpha_{1}$.

Proposition 2: The mean $\mu_{\triangle_{1}}$ and variance $\sigma_{\triangle_{1}}^{2}$ in Theorem 2 are

$$
\frac{a}{\mu_{\varepsilon_{2}}}\left(1-\frac{m}{a \mu_{\varepsilon_{2}}}+\frac{s}{\mu_{\varepsilon_{2}}^{2}}\right) \text { and } \frac{a^{2}}{\mu_{\varepsilon_{2}}^{2}}\left(\frac{t}{a^{2}}+\frac{s}{\mu_{\varepsilon_{2}}^{2}}-\frac{2 m}{a \mu_{\varepsilon_{2}}}\right) \text {, }
$$

respectively, where $a \triangleq \mu^{\mathrm{H}} \mathbf{P} \mu+\operatorname{tr}(\Sigma \mathbf{P}), s \triangleq 4 \mu^{\mathrm{H}} \mathbf{P} \Sigma \mathbf{P} \mu+2 \operatorname{tr}(\Sigma \mathbf{P})^{2}, t \triangleq 4 \mu^{\mathrm{H}} \mathbf{Q} \Sigma \mathbf{Q} \mu+2 \operatorname{tr}(\Sigma \mathbf{Q})^{2}$, and $m \triangleq 4 \mu^{\mathrm{H}} \mathbf{Q} \Sigma \mathbf{P} \mu+2 \operatorname{tr}(\mathbf{Q} \Sigma \mathbf{P} \Sigma)$.

Similarly, directly finding the optimal $\alpha_{2}$ from calculating the outage probability in (19) is intractable. The main idea of the proposed method to find the semi-analytical solution of $\alpha_{2}$ is described as follows. We first rearrange CR's rate formula $R\left(H_{21}, H_{22}\right)$ into a weighted sum of non-central chi-square random variables which can be further approximated by a single chisquare random variable. Then we can semi-analytically find $\alpha_{2}$ minimizing the outage probability $P_{\text {out }}^{C R}$ with the given outage capacity $R^{C R}$. This is summarized in the following theorem. Note that both $v\left(\alpha_{2}\right)$ and $w\left(\alpha_{2}\right)$ are simple rational functions of $\alpha_{2}$.

Theorem 3: The precoding coefficient $\alpha_{2}$ for the slow fading CR channel can be found by solving

$$
\min _{\alpha_{2}} \frac{\gamma\left(\frac{w\left(\alpha_{2}\right)}{2}, \frac{c d-1}{2 v\left(\alpha_{2}\right)}\right)}{\Gamma\left(\frac{w\left(\alpha_{2}\right)}{2}\right)}
$$

where $d=\left(2^{R^{C R}}-1\right) / \sigma_{\hat{x}_{c}}^{2}, \Gamma$ is the gamma function, $\gamma(.,$.$) is the incomplete gamma function,$ and

$$
v\left(\alpha_{2}\right)=\frac{1}{2} \frac{\operatorname{tr}(\Sigma \mathbf{E} \Sigma \mathbf{E})+2 \mu^{\mathrm{H}} \mathbf{E} \Sigma \mathbf{E} \mu}{\operatorname{tr}(\Sigma \mathbf{E})+\mu^{\mathrm{H}} \mathbf{E} \mu}, w\left(\alpha_{2}\right)=2 \frac{\left(\operatorname{tr}(\Sigma \mathbf{E})+\mu^{\mathrm{H}} \mathbf{E} \mu\right)^{2}}{\operatorname{tr}(\Sigma \mathbf{E} \Sigma \mathbf{E})+2 \mu^{\mathrm{H}} \mathbf{E} \Sigma \mathbf{E} \mu},
$$


where $\mathbf{E}=\left(1-c_{0} d\right)(\mathbf{P}+\mathbf{Q})+d \mathbf{D}$, and $c, c_{0}$ are defined right after (15).

The problem of solving (24) can be further approximated by the following corollary.

Corollary 1: The precoding coefficient $\alpha_{2}$ for the slow fading CR channel can be approximately found by solving

$$
\min _{\alpha_{2}}\left(1-\exp \left(-\frac{s(c d-1)}{2 v\left(\alpha_{2}\right)}\right)\right)^{\frac{w\left(\alpha_{2}\right)}{2}}
$$

where

$$
s= \begin{cases}1, & 0<w\left(\alpha_{2}\right)<2 \\ \left(\Gamma\left(1+w\left(\alpha_{2}\right) / 2\right)\right)^{\frac{-2}{w\left(\alpha_{2}\right)},} & w\left(\alpha_{2}\right)>2 .\end{cases}
$$

Note that Corollary 1 does not need the computation of the incomplete gamma function in (24), thus the search of $\alpha_{2}$ becomes simpler.

\section{ASYMPTOTIC ANALYSIS}

Since a Rician fading channel becomes non-fading when its $K$-factor approaches infinity, and the relaying ratio and precoding coefficient are well known for non-fading channels [4], we can verify the correctness of the proposed methods by making the $K$-factor infinity.

\section{A. Asymptotic analysis of $\alpha_{1}$}

In the high $K$-factor region, the channel gains are almost deterministic and knowing the mean values of the channels is almost the same as knowing the channel gains. With the assumption that $\left|\mu_{i j}\right|^{2}+\sigma_{i j}^{2}=1,1 \leq i, j \leq 2$, parameters and performances of slow and fast fading channels should converge to that of the non-fading channel with unity channel gain. Thus both $\sigma_{\varepsilon_{1}}$ and $\sigma_{\triangle_{1}}$ approach zero. Besides,

$$
\lim _{K \rightarrow \infty} \mu_{\varepsilon_{1}}=\varepsilon_{1}, \lim _{K \rightarrow \infty} \mu_{\varepsilon_{2}}=\varepsilon_{2},
$$

where the definitions of $\varepsilon_{1}$ and $\varepsilon_{2}$ are given right after (43). For the fast fading case, the left hand side of (16) becomes

$$
\lim _{K \rightarrow \infty} E\left[R\left(H_{11}, H_{12}\right)\right]=\log \left(\frac{1+\mu_{\varepsilon_{1}}}{1+\mu_{\varepsilon_{2}}}\right)=\log \left(\frac{1+\varepsilon_{1}}{1+\varepsilon_{2}}\right)=\log \left(1+\triangle_{1}^{-1}\right)=\log \left(1+P_{p}\right),
$$

where $\triangle_{1}$ is defined in (21). On the other hand, for the slow fading case (22) becomes

$$
\lim _{K \rightarrow \infty}\left(\mu_{\triangle_{1}}+\sqrt{\frac{r}{P_{\text {out }}^{P}}-1} \sigma_{\triangle_{1}}\right)=\lim _{K \rightarrow \infty} \mu_{\triangle_{1}}=\triangle_{1}=P_{p}^{-1} .
$$

From (28) and (29) we can find that $\alpha_{1}$ converges in both fast and slow fading scenarios to the same value. From the definition of $\triangle_{1}$ we have

$$
\frac{P_{p}+\alpha_{1} P_{c}+2 \sqrt{\alpha_{1} P_{c} P_{p}}}{\left(1-\alpha_{1}\right) P_{c}+1}=P_{p}
$$

which results in the same $\alpha_{1}$ as that in the non-fading CR case [4]. Thus we can conclude that the $\alpha_{1}$ 's found by the proposed methods in both fast and slow fading channels converge to that of the non-fading case when $K \rightarrow \infty$. 
B. Asymptotic analysis of $\alpha_{2}$

Again the precoding coefficient $\alpha_{2}$ should coincide with that of the non-fading case when $K \rightarrow \infty$. In the following we verify this statement.

1) Fast fading: When $K \rightarrow \infty$, both $\mu_{22}$ and $\mu_{21}$ approach 1, and (18) becomes

$$
\lim _{K \rightarrow \infty} \alpha_{2}=\frac{\left(1+\sqrt{\frac{\alpha_{1} P_{c}}{P_{p}}}\right)\left(1-\alpha_{1}\right) P_{c}}{\left(1-\alpha_{1}\right) P_{c}+1}=\left(1+\sqrt{\frac{\alpha_{1} P_{c}}{P_{p}}}\right) \alpha_{2}^{M M S E},
$$

where $\sqrt{\frac{\alpha_{1} P_{c}}{P_{p}}}$ is due to the CR's relaying of the primary signal and $\alpha_{2}^{M M S E}$ is the precoding coefficient of the non-fading case [4]. Thus it is verified that the proposed method also results in the MMSE linear assignment as the perfect CSIT case when $K \rightarrow \infty$.

2) Slow fading: The asymptotic property of $\alpha_{2}$ is described in the following corollary with proof given in Appendix $\mathrm{E}$

Corollary 2: When $K$-factor $\rightarrow \infty, \alpha_{2}$ derived in Theorem 3 converges to $\left(1+\sqrt{\frac{\alpha_{1} P_{c}}{P_{p}}}\right) \alpha_{2}^{M M S E}$.

From (30) and Corollary 2, we conclude that $\alpha_{2}$ 's for slow and fast fading channels converge to the same value in [4] when the $K$-factor $\rightarrow \infty$.

\section{Practical Lattice-BASED Linear-AssignMent Gel'FAnd-Pinsker CODing}

All the previous results are based on the LA-GPC with theoretical, unstructured Gaussian random codebooks. In the following we introduce a practical lattice-based coding to implement the LA-GPC for the CR user. With the carefully designed filters specified in Theorem 4 , the proposed lattice-based LA-GPC is a non-trivial extension of the well-known lattice-based DPC [14]. First, the side-information filter is selected according to the aforementioned precoding coefficient $\alpha_{2}$ instead of directly using the MMSE filter $\alpha_{c}$ described after (6) for the interferencefree channel as in Section II-B of [14]. Secondly, with only the statistics of the CSIT, the receiver filter selection is more involved than simply choosing it as $\alpha_{c}$ in DPC [14] [15]. Specifically, this filter must be different from the side-information filter, and this is contrary to the common practice in the lattice-based DPC [14] [15]. Finally, we adopt the finite dimensional self-similar lattices to implement the lattice coding structure which is much more feasible than the latticebased DPC in [14] using a very long codeword length. The proposed coding works very well with a reasonable codeword length (and decoding latency), and our simulation in Section VI-C verifies this.

To illustrate the lattice-based LA-GPC, we focus on the following channel corresponding to (2) with discrete time index $t$ as

$$
Y(t)=H_{x}(t) X_{L}(t)+H_{S}(t) S(t)+Z(t),
$$


where $1 \leq t \leq T, T$ is the codeword length. To emphasize the difference between lattice coding and random Gaussian codebooks used in the previous sections, we use $X_{L}(t)$ instead of $X(t)$ to represent the lattice-coded signal. As in Section II-C, we first focus on the rate (5) with given channel realizations $H_{x}(t)=h_{x}(t)$ and $H_{s}(t)=h_{s}(t)$. We also rewrite (31) in an equivalent real super channel to present the lattice coding more easily. By concatenating all $T$ symbols, the channel becomes

$$
\underline{Y}=\mathbf{H}_{\mathbf{x}} \underline{X}_{L}+\mathbf{H}_{\mathbf{s}} \underline{S}+\underline{Z},
$$

where $\underline{X}_{L}=\left(\underline{X}_{L, 1}^{\mathrm{T}}, \ldots, \underline{X}_{L, \mathrm{~T}}^{\mathrm{T}}\right)^{\mathrm{T}}$ and $\underline{X}_{L, t}=\left(\operatorname{Re}\left\{X_{L}(t)\right\}, \operatorname{Im}\left\{X_{L}(t)\right\}\right)^{\mathrm{T}}$. The non-causally known sideinformation vector at the transmitter $\underline{S}$ and the noise vector $\underline{Z}$ are obtained similarly from $S(t)$ and $Z(t)$, respectively, as $\underline{X}_{L}$ from $X_{L}(t)$. The covariance matrices of $\underline{S}$ and $\underline{Z}$ are denoted by $\Sigma_{s}$ and $\Sigma_{z}$, respectively. The $2 T \times 2 T$ block-diagonal real channel matrix $\mathbf{H}_{\mathbf{x}}$ is $\operatorname{diag}\left(\left[\mathbf{H}_{\mathbf{x}}{ }^{1} \cdots \mathbf{H}_{\mathbf{x}}{ }^{T}\right]\right)$, where the $t$ th diagonal term is

$$
\mathbf{H}_{\mathbf{x}}{ }^{t}=\left[\begin{array}{cc}
\operatorname{Re}\left\{h_{x}(t)\right\} & -\operatorname{Im}\left\{h_{x}(t)\right\} \\
\operatorname{Im}\left\{h_{x}(t)\right\} & \operatorname{Re}\left\{h_{x}(t)\right\}
\end{array}\right],
$$

and $\mathbf{H}_{\mathbf{s}}$ is formed from $H_{s}(t)$ in the same way as $\mathbf{H}_{\mathbf{x}}$ from $H_{x}(t)$. The channel input power constraint is $\left(1-\alpha_{1}\right) P_{c} / 2$ because the CR user transmits its own signal with power $P_{c} / 2$ per real dimension, and relays the primary user's signal with power $\alpha_{1} P_{c} / 2$.

We will first give a brief review of the lattice codebook, then introduce our proposed lattice coding. A $2 T$-dimension real lattice $\Lambda$ is defined as $\Lambda=\left\{\mathbf{G b}: \mathbf{b} \in \mathbb{Z}^{2 T}\right\}$, where $\mathbf{G}$ is the $2 T \times 2 T$ generator matrix of $\Lambda$. The Voronoi region $\mathcal{V}$ is the set of points $\mathbf{g} \in \mathbb{R}^{2 T}$ which are closest to $\mathbf{0}$ in Euclidean distance than to other lattice points $\lambda \in \Lambda$. Every $\mathbf{g} \in \mathbb{R}^{2 T}$ can be uniquely written as $\mathbf{g}=\lambda+\mathbf{n}_{g}$, where $\lambda \in \Lambda$ and $\mathbf{n}_{g} \in \mathcal{V}$. With quantizer input $\mathbf{g}$, the lattice quantizer associated with $\mathcal{V}$ is defined as $Q(\mathbf{g})=\lambda$, if $\mathbf{g} \in \lambda+\mathcal{V}$. The modulo- $\Lambda$ operation associated with $\mathcal{V}$ is then

$$
\mathbf{g} \bmod \Lambda=\mathbf{g}-Q(\mathbf{g}) \text {. }
$$

We define the nested-lattice codes with length $2 T$ as

Definition 1: Let $\Lambda_{c}$ be a lattice and $\Lambda_{q}$ be a sublattice of it, that is, $\Lambda_{q} \subseteq \Lambda_{c}$. The codeword set of the nested lattice code is $\mathcal{C}_{c}=\left\{\Lambda_{c} \bmod \Lambda_{q}\right\} \triangleq\left\{\Lambda_{c} \cap \mathcal{V}_{q}\right\}$. The code rate of this nested lattice code is $R_{c}=\frac{1}{T} \log \left\|\mathcal{V}_{q}\right\| /\left\|\mathcal{V}_{c}\right\|$, where $\mathcal{V}_{c}$ and $\mathcal{V}_{q}$ are the fundamental Voronoi regions of $\Lambda_{c}$ and $\Lambda_{q}$, respectively. And for a bounded Jordan-measurable region $\mathcal{V} \subset \mathbb{R}^{2 T},\|\mathcal{V}\|$ denotes the volume of $\mathcal{V}$.

For conciseness of the paper, we focus on the slow fading channels where the channels remain constant within a codeword length. The proposed scheme can be easily modified for the fast fading channels [16]. The proposed coding works as follows

Encoder: The encoder selects a codeword $\mathbf{c}_{\mathbf{c}} \in \mathcal{C}_{c}$ according to the message index and sends

$$
\underline{X}_{L}=\sqrt{\left(1-\alpha_{1}\right) P_{c}}\left(\left(\mathbf{c}_{\mathbf{c}}-\mathbf{F}_{\mathbf{s}} \underline{S}-\underline{D}\right) \bmod \Lambda_{q}\right),
$$


where the $2 T \times 2 T$ side-information filter $\mathbf{F}_{\mathbf{s}}$ is formed from $\alpha_{2}$ in Theorem 3 as

$$
\mathbf{I}_{T} \otimes\left[\begin{array}{cc}
\operatorname{Re}\left\{\alpha_{2}\right\} & -\operatorname{Im}\left\{\alpha_{2}\right\} \\
\operatorname{Im}\left\{\alpha_{2}\right\} & \operatorname{Re}\left\{\alpha_{2}\right\}
\end{array}\right],
$$

and $\otimes$ denotes the Kronecker product. The dither signal $\underline{D}$, uniformly distributed in $\mathcal{V}_{q}$ and independent of the channel, is known to both the transmitter and the receiver. This dither plays a critical role in making $\underline{X}_{L}$ independent of $\mathbf{c}_{\mathbf{c}}$ and $\underline{S}$ [16]. The second moment $P\left(\mathcal{V}_{q}\right)$ [15] of $\Lambda_{q}$ is set $1 / 2$ to satisfy the power constraint $\left(1-\alpha_{1}\right) P_{c} / 2$.

Decoder: The decoder performs the following operation on $\underline{Y}$

$$
\underline{\hat{Y}}=\mathbf{L}\left(\mathbf{F}_{\mathbf{r}} \underline{Y}+\underline{D}\right) \text {. }
$$

After some manipulations [16], $\underline{\hat{Y}}$ in (36) can be rewritten as

$$
\underline{\hat{Y}}=\mathbf{L}\left(\mathbf{c}_{\mathbf{c}}^{\prime}\right)+\mathbf{e},
$$

where $\mathbf{c}_{\mathbf{c}}^{\prime} \in \Lambda_{q}+\mathbf{c}_{\mathbf{c}} \subset \Lambda_{c}$ and

$$
\mathbf{e} \triangleq\left(\mathbf{F}_{\mathbf{r}} \tilde{\mathbf{H}}-\mathbf{I}_{2 T}\right) \underline{D}+\left(\mathbf{F}_{\mathbf{r}} \mathbf{H}_{\mathbf{s}}-\mathbf{F}_{\mathbf{s}}\right) \underline{S}+\mathbf{F}_{\mathbf{r}} \underline{Z},
$$

where $\tilde{\mathbf{H}}=\sqrt{\left(1-\alpha_{1}\right) P_{c}} \mathbf{H}_{x}$. The receiver filter $\mathbf{F}_{\mathbf{r}}$ is obtained according to the MMSE filter for estimating the auxiliary random variable $U$ in (6) from $Y$ in (2) as [16] [17]

$$
\mathbf{F}_{\mathbf{r}}=\left(\frac{1}{2} \tilde{\mathbf{H}}^{\mathrm{T}}+\mathbf{W} \Sigma_{s} \mathbf{H}_{\mathbf{s}}^{\mathrm{T}}\right)\left(\frac{1}{2} \tilde{\mathbf{H}} \tilde{\mathbf{H}}^{\mathrm{T}}+\mathbf{H}_{\mathbf{s}} \Sigma_{s} \mathbf{H}_{\mathbf{s}}^{\mathrm{T}}+\Sigma_{z}\right)^{-1},
$$

where $\Sigma_{s}$ is the covariance matrix of $\underline{S}$. The whitening matrix filter $\mathbf{L}$ for e must satisfy

$$
\mathbf{L}^{\mathrm{T}} \mathbf{L}=\Sigma_{E}^{-1}
$$

where $\Sigma_{E}$ is the covariance matrix of e when $T \rightarrow \infty$ as

$$
\Sigma_{E}=\frac{1}{2}\left(\mathbf{F}_{\mathbf{r}} \tilde{\mathbf{H}}-\mathbf{I}_{2 T}\right)\left(\mathbf{F}_{\mathbf{r}} \tilde{\mathbf{H}}-\mathbf{I}_{2 T}\right)^{\mathrm{T}}+\left(\mathbf{F}_{\mathbf{r}} \mathbf{H}_{\mathbf{s}}-\mathbf{F}_{\mathbf{s}}\right) \Sigma_{S}\left(\mathbf{F}_{\mathbf{r}} \mathbf{H}_{\mathbf{s}}-\mathbf{F}_{\mathbf{s}}\right)^{\mathrm{T}}+\mathbf{F}_{\mathbf{r}} \Sigma_{z} \mathbf{F}_{\mathbf{r}}^{\mathrm{T}} .
$$

Then we can use the generalized minimum Euclidean distance lattice decoder to decode $\mathbf{c}_{\mathbf{c}}$. First the decoder finds

$$
\hat{\mathbf{b}}=\arg \min \left|\hat{\mathbf{y}}-\mathbf{L G}_{c} \mathbf{b}\right|^{2}
$$

where $\mathbf{G}_{c}$ is the generator matrix of the channel coding lattice $\Lambda_{c}$. After that the decoded codeword is $\hat{\mathbf{c}}_{c}=\left[\mathbf{G}_{c} \hat{\mathbf{b}}\right] \bmod \Lambda_{q}$.

With "good nested lattices" defined in [15], we have the following result. The proof is omitted and can be found in [16].

Theorem 4: Let the side-information filter $\mathbf{F}_{\mathbf{s}}$, receiver filter $\mathbf{F}_{\mathbf{r}}$, and whitening filter $\mathbf{L}$ be selected as (35), (39), and (40), respectively. Based on sequences of good nested lattices, the 
coding specified in (34)-(42) with (3) is able to achieve the linear-assignment rate $R\left(h_{22}, h_{21}\right)=$ $\frac{1}{T} \log \left|\Sigma_{E}\right|^{-1}$ under power constraint $\left(1-\alpha_{1}\right) P_{c}$ when $T \rightarrow \infty$ where $\Sigma_{E}$ is defined in (41).

It can be proved that with full CSIT, the proposed coding reduces to the DPC in [14] [15]. And according to our simulation, the DPC suffers severely when it is naively applied to channels without perfect CSIT.

\section{Simulation RESUlts}

In this section we demonstrate the performance of the proposed LA-GPC based CR system under both fast and slow fading channels. We also show the performance of the proposed practical lattice encoder/decoder which implements the LA-GPC. For simplicity, the four channels in Fig. 1 are assumed independent Rician with the same $K$-factors.

\section{A. Fast fading}

To verify the goodness of the proposed approximation, we first compare it with brute-force full search in terms of the primary user's rate. Signal and noise powers are set as $P_{c}=P_{p}=10$ and $\sigma_{Z_{p}}^{2}=\sigma_{Z_{s}}^{2}=1$. This comparison is shown in Fig. 2. The unit of the vertical axis is bits per channel use, bpcu. We can see that the proposed approximation performs well for all $K$-factors. That is to say, the primary user's rate is approximately the same as that with $\alpha_{1}$ obtained by brute-force search. More specifically, the proposed method may over-design $\alpha_{1}$ slightly at the small $K$-factor region such that the primary user's rate becomes larger than the target. At the large $K$-factor region, $\alpha_{1}$ obtained by the proposed method almost coincides with that obtained by full search. The CR user's ergodic capacities using $\alpha_{1}$ and $\alpha_{2}$ from the proposed method is shown in Fig. 3, It can be observed that the over-designed $\alpha_{1}$ slightly increases the primary's rate at the cost of the CR user's rate. However, since $\alpha_{1}$ is only slightly over-designed, the resulting change of the $\mathrm{CR}$ user's rate is small. In addition, we also compare cases of treating interference as noise and with full CSIT. The former neglects any useful information to cancel the interference, and incurs a huge rate loss for all $K$-factors considered. The ergodic capacities for the partial and full CSIT cases converge when the $K$-factor is large. This phenomenon has been verified in Section IV. The rate loss at the low $K$-factor region is due to two factors. One is the imperfect CSIT. The other is that the linear assignment strategy may not be optimal for the partial CSIT case. Until now, the optimal strategy for the partial CSIT case is still an open problem. Note that all the LA-GPC curves can also be obtained from the lattice-code achievable rate $1 / T \log \left|\Sigma_{E}\right|^{-1}$ with filters specified in Theorem 4 and $T \rightarrow \infty$. The naive DPC scheme assumes that the channels always take values at their means, and uses these mean values to design the DPC filter. That is, the $\mathbf{F}_{\mathbf{s}}$ filter is naively chosen according to $\alpha_{c}$ in Section II-B with $H_{x}$ replaced by $E\left[H_{x}\right]$, and $\mathbf{F}_{\mathbf{r}}=\mathbf{F}_{\mathbf{S}} \mathbf{H}_{\mathbf{x}}{ }^{-1}$. It can be observed that the naive DPC scheme has a significant rate loss compared to the proposed method in a wide range of $\mathrm{K}$-factors. As 
expected, the ergodic capacities of all cases except treating interference as noise converge to the same value when the $K$-factor is large.

\section{B. Slow fading}

We first demonstrate the effectiveness of the approximation for $\alpha_{1}$ in terms of the primary user's outage capacity. A good $\alpha_{1}$ will make $P\left(R\left(H_{11}, H_{21}\right)<R^{P}\right)$ as close to $P_{\text {out }}^{P}$ as possible. We then compare the values of $\alpha_{2}$ obtained from (24) and full search. Finally, we illustrate the CR user's outage probabilities with $\alpha_{2}$ 's from these two methods. Signal and noise powers are set as $P_{c}=P_{p}=10$ and $\sigma_{Z_{p}}^{2}=\sigma_{Z_{s}}^{2}=1$. Since different channel conditions can support different outage capacity and outage probability pairs, it is more reasonable to consider the supportable pairs than using the same pair for different $K$-factors. Thus, in the following, we set primary user's outage capacity (in bpcu) and probability pairs as (1, 0.1), (2, 0.1), (2, 0.01), and (2, 0.01 ) for $K$-factors of $0,5,10$, and $15 \mathrm{~dB}$, respectively. Moreover, the CR user's target rates are set as $0.2,0.5,1$, and $1.5 \mathrm{bpcu}$, respectively. Fig. 4 checks whether the primary user's performance constraint (20) is satisfied. Compared to the baseline case without the presence of the CR transmitter, we find that the approximation of $\alpha_{1}$ is very tight in the low $K$-factor region, and is good for medium and high $K$-factors. The tight approximation is a result of the $K$-factor dependent $r$. When the $K$-factor is 0 or $5 \mathrm{~dB}$, it can be found that $\delta \geq 0$ and the distribution of $\triangle_{1} f_{\triangle_{1}}$ is asymmetric. Thus we let $r=1$. On the other hand, when the $K$-factor is 10 or $15 \mathrm{~dB}$, $f_{\triangle_{1}}$ is unimodal and symmetric simultaneously. In addition, $\delta \geq 2 / \sqrt{3}$. Thus we set $r=2 / 9$ to further tighten the original Cantelli's inequality. The slightly over-designed $\alpha_{1}$ ensures that the primary user's outage probability constraint is met at the cost of the CR user's rate.

The CR user's outage probabilities with $\alpha_{1}$ and $\alpha_{2}$ both obtained from approximations and full searches are given in Fig. 5. Recall that $\alpha_{1}$ is slightly over-designed at medium and high $K$-factors. This reduces the CR's power for its own signal, and increases the outage probability. On the other hand, when the $K$-factor is small, the worse channel condition dominates the performance. In addition, we can find that the approximation from Alzer's bound [?] of the incomplete gamma function is good for the considered $K$-factors. Naive DPC again incurs a large rate loss in the low to medium $K$-factor region. It can be seen that if the interference is treated as noise, the outage probability is much worse than those of the proposed precoding methods. Similar to the fast fading case, there is a gap between the outage probabilities of the full and partial CSIT cases. Since the optimal precoding strategy is still an open problem, the minimum possible gap is unknown. The non-decreasing outage probability curves of treating interference as noise and full CSIT are due to the facts that the considered target rates are not identical for different $K$-factors. 


\section{Lattice implementation with finite codelength}

We investigate the error performance of lattice precoding with a reasonable codeword length (and decoding latency) at large enough SNR. A pair of self-similar nested lattices is used, with the Gosset lattice $E_{8}$ [18], which has the densest packing in 8-dimension, as the fine lattice $\Lambda_{c}$. The reason to use the Gosset lattice $E_{8}$ is that in moderate dimensions, it is well-known that the best lattices in terms of coding gain are also good quantizers. That is, the lattice can also be good $\Lambda_{q}$. The coarse lattice $\Lambda_{q}$ is generated by $\Lambda_{q}=Q \Lambda_{c}$ where the coding rate is given by $R=2 \log Q$. Recall that transmitted signals is assumed to be Gaussian in previous derivations. The Gaussianity of the signals at the proposed lattice-based CR transmitter output with a reasonably short codeword length $T=4$ is shown in Fig. 6, It is clear that the transmitter output distribution is almost Gaussian.

In the simulation of codeword error probabilities we consider two $K$-factors: $0 \mathrm{~dB}$ and $10 \mathrm{~dB}$ and two code rates: 2 and 4 bits per channel use. The noise variance is normalized to 1 and the primary user's power constraint is assumed to be $P_{p}=100$. The LA-GPC curves are obtained from the lattice-code achievable rate with filters specified in Theorem 4 . A Fano sequentialdecoding based lattice decoder [19] is used to solve (42). We compare the lattice coding results with the theoretical results with random Gaussian codebooks. The basis for the comparison is that the codeword error probability is approximately the same as the outage probability. The results are shown in Fig. 7 and Fig. 8. For comparison, we also show the results without interference from the primary user and treating interference as Gaussian noise. When the interference is treated as noise, the codeword error rate is 1 regardless of the SNR and $K$-factor ranges. It can be seen that in the considered $K$-factor range, the performance of the proposed nested lattice decoding is approximately the same as that derived theoretically. This verifies the goodness of the used Gosset lattice code. We also observe that in Fig. 7 with $K$-factor $=0 \mathrm{~dB}$, lattice precoding with partial CSIT performs slightly better than the theoretical outage result. A similar phenomenon was also reported in [20].

\section{CONCLUSION}

In this paper we considered the cognitive radio channel with partial CSIT. Using the linearassignment Gel'fand-Pinsker coding, we proposed semi-analytical methods for finding the relaying ratios and the precoding coefficients for both fast and slow fading channels. Asymptotic analysis showed that the relaying ratios and the precoding coefficients obtained by the proposed methods converge to those with full CSIT when the $K$-factor approaches infinity. Simulation results showed that the proposed semi-analytical parameter design methods perform almost as well as exhaustive search. We also used nested-lattice coding and decoding to realize the precoding in practice. Simulation results showed that the nested-lattice coding scheme can effectively reach the achievable rate of the CR channel with partial CSIT at high SNR. 


\section{APPENDIX}

\section{A. Proof of Theorem 1$]$}

After applying the expectation operation to (14) we have

$$
\begin{aligned}
E\left[R\left(H_{11}, H_{12}\right)\right] & =E\left[\log \left(1+\underline{H}_{p}^{H} \mathbf{S} \underline{H}_{p}\right)\right]-E\left[\log \left(1+\underline{H}_{p}^{H} \mathbf{Q} \underline{H}_{p}\right)\right] \\
& \triangleq E\left[\log \left(1+\varepsilon_{1}\right)\right]-E\left[\log \left(1+\varepsilon_{2}\right)\right]
\end{aligned}
$$

where $\mathbf{S} \triangleq \mathbf{P}+\mathbf{Q}, \varepsilon_{1} \triangleq \underline{H}_{p}^{H} \mathbf{S} \underline{H}_{p}$, and $\varepsilon_{2} \triangleq \underline{H}_{p}^{H} \mathbf{Q} \underline{H}_{p}$. To guarantee that (11) is valid, we expand $f\left(\varepsilon_{1}\right) \triangleq \log \left(1+\varepsilon_{1}\right)$ and $g\left(\varepsilon_{2}\right) \triangleq \log \left(1+\varepsilon_{2}\right)$ by the Taylor series with different orders, respectively. The reason is as the following. We first expand $\log \left(1+\varepsilon_{1}\right)$ by the $k$-th order Taylor series respect to the mean of $\varepsilon_{1}, \mu_{\varepsilon_{1}}$, as

$$
\log \left(1+\varepsilon_{1}\right)=\log \left(1+\mu_{\varepsilon_{1}}\right)-\log e \sum_{n=2}^{k} \frac{(-1)^{n}}{n} \frac{\left(\varepsilon_{1}-\mu_{\varepsilon_{1}}\right)^{n}}{\left(1+\mu_{\varepsilon_{1}}\right)^{n}}+o_{1} \triangleq f_{k}\left(\varepsilon_{1}\right)+o_{1},
$$

where $f_{k}\left(\varepsilon_{1}\right)$ is the truncated $k$-th order Taylor expansion and $o_{1}$ is the Lagrange remainder. After applying the expectation operator to (44) and truncating the remainder $o$ we have

$$
E\left[f_{k}\right]=\log \left(1+\mu_{\varepsilon_{1}}\right)-\log e \Sigma_{n=2}^{k} \frac{(-1)^{n}}{n} \frac{E\left[\left(\varepsilon_{1}-\mu_{\varepsilon_{1}}\right)^{n}\right]}{\left(1+\mu_{\varepsilon_{1}}\right)^{n}},
$$

where $E\left[f_{k}\left(\varepsilon_{1}\right)\right]$ is further simplified as $E\left[f_{k}\right]$. Since the Taylor expansion of the logarithmic function $f$ is an alternating series, and $E\left[\left(\varepsilon_{1}-\mu_{\varepsilon_{1}}\right)^{n+1}\right]>0, \forall n \in \mathbb{N}$, from [11], we have the following relations: $E\left[f_{1}\right]>E\left[f_{3}\right]>E\left[f_{5}\right]>\cdots>E[f]$ and $E\left[f_{2}\right]<E\left[f_{4}\right]<E\left[f_{6}\right]<\cdots<E[f]$. Similar properties can be found for $g\left(\varepsilon_{2}\right)$. The truncated first order Taylor expansion of $E[\log (1+$ $\left.\left.\varepsilon_{2}\right)\right]$ can be derived in the same way. From the above we know that $E[f]-E[g]>E\left[f_{2 i}\right]-E\left[g_{2 j-1}\right]$. Thus solving the following equality

$$
E\left[f_{2 i}\right]-E\left[g_{2 j-1}\right]=R_{\text {ergodic }}^{P}
$$

makes the constraint (11) valid where $i, j \in \mathbb{N}$. Here we choose $i=j=1$ for simplicity. After some manipulations we have (16), where $\mu_{\varepsilon_{1}}, \sigma_{\varepsilon_{1}}^{2}$, and $\mu_{\varepsilon_{2}}$, the mean, variances of $\varepsilon_{1}$, and the mean of $\varepsilon_{2}$, can be found according to [11].

\section{B. Proof of Proposition 1}

With the fact that $c_{0}(\mathbf{P}+\mathbf{Q})-\mathbf{D}$ is positive semi-definite and $c>0, B\left(\alpha_{2}\right)$ is positive. Then we can generalize the result in [21], which is aimed for the ratio of quadratic forms, to the ratio of general quadratic forms in (17). Therefore, we have

$$
E\left[\frac{B^{\prime}\left(\alpha_{2}\right)}{B\left(\alpha_{2}\right)}\right] \simeq \frac{E\left[B^{\prime}\left(\alpha_{2}\right)\right]}{E\left[B\left(\alpha_{2}\right)\right]}=0
$$

\footnotetext{
$\dagger$ "General" means that there is not only the term of the quadratic form, but also terms of lower orders.
} 
Since $B\left(\alpha_{2}\right)$ is a second order polynomial of $\alpha_{2}$, to proceed, we only need to consider the numerator, that is,

$$
E\left[B^{\prime}\left(\alpha_{2}\right)\right]=0
$$

With the fact that $\sigma_{22}^{2}+\left|\mu_{22}\right|^{2}=1$, the resulting precoding coefficient with the statistics of CSIT is as (18).

\section{Proof of Theorem 2}

From (14), (20) and (21), the optimal $\alpha_{2}$ will make

$$
P\left(\triangle_{1} \geq \frac{1}{2^{R^{P}}-1}\right)=P_{\text {out }}^{P} \text {. }
$$

To make this problem analytically tractable, we relax the equality constraint by inequality to find the sub-optimal solution. With the non-negativity of the transmission rate, the Cantelli's inequality [22] is a good candidate for finding the solution. Considering additional properties of the distribution $f_{\triangle_{1}}$, such as the modality and symmetry, the Cantelli's inequality can be further tightened [12]. Thus we propose the use of the following modified Cantelli's inequality to relax (46) as

$$
P\left(\triangle_{1} \geq \mu_{\triangle_{1}}+\delta \sigma_{\triangle_{1}}\right) \leq \frac{r}{1+\delta^{2}}
$$

According to different $f_{\triangle_{1}}$ resulting from different channel conditions, $r$ in (47) can be categorized as [12]

$$
r=\left\{\begin{array}{l}
1, \text { if } \delta \geq 0, \\
\frac{2}{9}, \text { if } \delta \geq \frac{2}{\sqrt{3}} \text { and } f_{\triangle_{1}} \text { is unimodal and symmetric. }
\end{array}\right.
$$

Comparing the left hand side of the inequalities in (46) and (47) results in

$$
\mu_{\triangle_{1}}+\delta \sigma_{\triangle_{1}}=\frac{1}{2^{R^{P}}-1}
$$

Also by comparing the right hand side of the inequalities in (46) and (47) we have $\delta=$ $\sqrt{r / P_{\text {out }}^{P}-1}$. Substituting $\delta$ into (49), we have (22). To determine $r$, we can simply substitute $r=1$ or $r=2 / 9$ to see whether the resulting $\delta$ meets the constraints (48). The constraints of modality and (or) symmetry which may be satisfied under different channel conditions are discussed in Section VI. Thus we can solve the outage probability constraint by finding $\alpha_{1}$ which satisfies the equality in (22). We now discuss the validity of (20). As for the effect of the modified Cantelli's inequality, note that the modified Cantelli's upper bound is larger than the real probability value [12]. Since we equate the upper bound to the target outage probability to solve $\alpha_{1}$, the resulting $\alpha_{1}$ will be larger than the optimal one from (46). 


\section{Proof of Theorem 3}

First, substituting (15) into (19), and after some manipulations we have the following optimization problem

$$
\min _{\alpha_{2}} P\left(z \triangleq \underline{H}_{c}^{\mathrm{H}} \mathbf{E} \underline{H}_{c}=\underline{H}_{2}^{\mathrm{H}} \mathbf{E}_{2} \underline{H}_{2}<c d-1\right),
$$

where $\mathbf{E}_{2}=\Sigma^{1 / 2} \mathbf{E} \Sigma^{1 / 2}$, and $\underline{H}_{2} \sim \mathcal{C N}\left(\Sigma^{-1 / 2} \mu, \mathbf{I}_{2}\right)$. The transformation from $\mathbf{E}$ to $\mathbf{E}_{2}$ is to make $\underline{H}_{2}$ have an identity covariance matrix for the convenience of the following analysis. After substituting the eigen decomposition $\mathbf{E}_{2}=\mathbf{v}^{\mathrm{H}} \Lambda \mathbf{v}$ into $z$, we have

$$
z=\underline{H}_{3}^{\mathrm{H}} \underline{H}_{3} \sim \lambda_{1} \chi^{2}\left(2,2\left|\mu_{31}\right|^{2}\right)+\lambda_{2} \chi^{2}\left(2,2\left|\mu_{32}\right|^{2}\right),
$$

where $\underline{H}_{3} \sim \mathcal{C N}\left(\mu_{3}, \mathbf{I}_{2}\right), \mu_{3}=\left[\mu_{31}, \mu_{32}\right]^{T} \triangleq \mathbf{v} \Sigma^{-1 / 2} \mu$, and $\lambda_{1}$ and $\lambda_{2}$ are the eigenvalues of $\mathbf{E}_{2}$. The notation $\chi^{2}(p, q)$ denotes the noncentral chi-square distribution with degree of freedom $p$ and non-centrality $q$. Note that the objective function (50) is a function of $\alpha_{2}$. That is, $\lambda_{1}, \lambda_{2}, \mu_{31}$, and $\mu_{32}$ are controlled by $\alpha_{2}$.

To calculate the outage probability (50), we resort to approximating $z$ by a scaled single central chi-square random variable [23] as

$$
z \sim \lambda_{1} \chi^{2}\left(2,2\left|\mu_{31}\right|^{2}\right)+\lambda_{2} \chi^{2}\left(2,2\left|\mu_{32}\right|^{2}\right) \simeq v \chi^{2}(w) .
$$

Recall that the chi-square distribution is a special case of the gamma distribution. Thus $w$ does not have to be an integer. The parameters $v$ and $w$ should be chosen such that both sides of (51) have the same first and second moments [23] as

$$
\begin{gathered}
v w=\lambda_{1}\left(1+\left|\mu_{31}\right|^{2}\right)+\lambda_{2}\left(1+\left|\mu_{32}\right|^{2}\right), \\
2 v^{2} w=\lambda_{1}^{2}\left(1+2\left|\mu_{31}\right|^{2}\right)+\lambda_{2}^{2}\left(1+2\left|\mu_{32}\right|^{2}\right) .
\end{gathered}
$$

With the fact

$$
\begin{aligned}
\lambda_{1}+\lambda_{2} & =\operatorname{tr}\left(\mathbf{E}_{2}\right)=\operatorname{tr}(\Sigma \mathbf{E}) \\
\lambda_{1}\left|\mu_{31}\right|^{2}+\lambda_{2}\left|\mu_{32}\right|^{2} & =\mu_{3}^{\mathrm{H}} \Lambda \mu_{3}=\mu^{\mathrm{H}} \Sigma^{\frac{-1}{2}} \mathbf{v}^{\mathrm{H}} \Lambda \mathbf{v} \Sigma^{\frac{-1}{2}} \mu=\mu^{\mathrm{H}} \mathbf{E} \mu
\end{aligned}
$$

we can find the value of $v w$. Similarly, from

$$
\begin{gathered}
\lambda_{1}^{2}+\lambda_{2}^{2}=\operatorname{tr}\left(\mathbf{E}_{2} \mathbf{E}_{2}\right)=\operatorname{tr}(\Sigma \mathbf{E} \Sigma \mathbf{E}) \\
, \lambda_{1}^{2}\left|\mu_{31}\right|^{2}+\lambda_{2}^{2}\left|\mu_{32}\right|^{2}=\mu^{\mathrm{H}} \Sigma^{\frac{-1}{2}} \mathbf{E}_{2} \mathbf{E}_{2} \Sigma^{\frac{-1}{2}} \mu=\mu^{\mathrm{H}} \mathbf{E} \Sigma \mathbf{E} \mu .
\end{gathered}
$$

we can find the value of $2 v^{2} w$. Then $v$ and $w$ can be expressed as (25). After that, solving (50) with (51) is equivalent to solving

$$
\min _{\alpha_{2}} \frac{\int_{0}^{\frac{c d-1}{2 v\left(\alpha_{2}\right)}} x^{\frac{w\left(\alpha_{2}\right)}{2}-1} e^{-t} d t}{\Gamma\left(\frac{w\left(\alpha_{2}\right)}{2}\right)}
$$

which can be further represented as (24). 


\section{E. Proof of Corollary 1}

From [?] we know that the incomplete gamma function can be bounded as following

$$
\left(1-e^{-s x}\right)^{a}<\frac{\gamma(a, x)}{\Gamma(a)}
$$

where

$$
s= \begin{cases}1, & 0<a<1 \\ (\Gamma(1+a))^{\frac{-1}{a}}, & a>1\end{cases}
$$

Let $a=\frac{w\left(\alpha_{2}\right)}{2}$ and $x=\frac{c d-1}{2 v\left(\alpha_{2}\right)}$, we then have (26).

\section{F. Proof of Corollary 2}

As the $K$-factor $\rightarrow \infty$, the distribution of $z$ defined in (50) becomes a delta function with a nonzero value at its mean $\mu_{z}$. In the following we need to find $\alpha_{2}$ maximizing the mean value of $z$ which can be approximated by $v w$, as shown in (51). Since $c$ in (50) is a function of $\alpha_{2}$, we must take it into consideration. The problem becomes to maximize $\mu_{z}-c d$. As both $\mu_{21}$ and $\mu_{22} \rightarrow 1$, and $\sigma_{21}^{2}$ and $\sigma_{22}^{2} \rightarrow 0$ at very high $K$-factor, from (52), (53), and (54), we have

$$
\lim _{K \rightarrow} \mu_{z}=v w=\mu^{\mathrm{H}} \mathbf{E} \mu+\operatorname{tr}(\Sigma \mathbf{E}) \simeq e_{11}+e_{12}+e_{21}+e_{22},
$$

where $e_{11}, e_{12}, e_{21}$, and $e_{22}$ are the four entries of $\mathbf{E}$. Since $\mu_{z}-c d$ is a quadratic formula of $\alpha_{2}$, we can take derivative to find the maximum as following

$$
\frac{1}{d} \frac{\partial}{\partial \alpha_{2}^{*}}\left(\mu_{z}-c d\right)=-\left(\left(1-\alpha_{1}\right) P_{c}+1\right) P_{p} \alpha_{2}+\left(P_{p}+\sqrt{\alpha_{1} P_{c} P_{p}}\right)\left(1-\alpha_{1}\right) P_{c}=0 .
$$

Then $\alpha_{2}$ can be found as

$$
\lim _{K \rightarrow \infty} \alpha_{2}=\frac{\left(1+\sqrt{\frac{\alpha_{1} P_{c}}{P_{p}}}\right)\left(1-\alpha_{1}\right) P_{c}}{\left(1-\alpha_{1}\right) P_{c}+1}=\left(1+\sqrt{\frac{\alpha_{1} P_{c}}{P_{p}}}\right) \alpha_{2}^{M M S E},
$$

which is the same as that of the non-fading case.

\section{REFERENCES}

[1] J. Mitola, "Cognitive radio: An integrated agent architecture for software defined radio," Ph.D. dissertation, KTH Royal Inst. Technology, Stockholm, Sweden, 2000.

[2] N. Devroye, P. Mitran, and V. Tarokh, "Achievable rates in cognitive radio channels," IEEE Trans. Inform. Theory, vol. 52, no. 5, pp. 1813-1827, May 2006.

[3] M. H. M. Costa, "Writing on dirty paper," IEEE Trans. Inform. Theory, vol. 29, pp. 439-441, May 1983.

[4] A. Jovicic and P. Viswanath, "Cognitive radio: An information-theoretic perspective," submitted to IEEE Trans. Inform. Theory, Apr. 2006.

[5] A. Bennatan and D. Burshtein, "On the fading paper achievable region of the fading MIMO broadcast channel," in 44th Annual Allerton Conference on Communication, Control and Computing, Sep. 2006. 
[6] S. I. Gelfand and M. S. Pinsker, "Coding for channel with random parameters," Problems of control and information theory, vol. 9, no. 1, pp. 19-31, 1980.

[7] D. Tse and P. Viswanath, Fundamentals of Wireless Communication. Cambridge University Press, 2005.

[8] J. Pinter, "Deterministic approximations of probability inequalities," Methods and Models of Operations Research, vol. 33, pp. 219-239, 1989.

[9] P.-H. Lin, C.-P. Lee, S.-C. Lin, and H.-J. Su, "Cognitive radio in slow fading channels with partial channel state information at the transmitter," in Proc. 42th Annual Conf. on Information Sciences and Systems (CISS2008), Princeton, N.J., USA, Mar. 19-21, 2008.

[10] P.-H. Lin, S.-C. Lin, and H.-J. Su, "Cognitive radio with partial channel state information at the transmitter," in Proc. IEEE Int. Conf. on Commun. (ICC 2008), Beijin, China, May, 19-23, 2008.

[11] A. M. Mathai and S. B. Provost, Quadratic forms in random variables. Marcel Dekker, New York, 1992.

[12] R. A. Ion, "Nonparametric statistical process control," Ph.D. dissertation, Wiskunde en Informatica, Universiteit van Amsterdam, Netherlands, 2001.

[13] M. S. Paollela, "Computing moments of ratios of quadratic forms in normal variables," Computational statistics and data analysis, vol. 42, pp. 313-331, 2003.

[14] R. Zamir, S. Shamai, and U. Erez, "Nested linear/lattice codes for structured multiterminal binning," IEEE Trans. Inform. Theory, vol. 48, no. 6, pp. 1250-1276, June 2002.

[15] H. E. Gamal, G. Caire, and M. O. Damen, "Lattice coding and decoding achieve the optimal diversity-vs-multiplexing tradeoff of MIMO channels," IEEE Trans. Inform. Theory, vol. 50, no. 6, pp. 968 - 985, Jun 2004.

[16] S.-C. Lin, P.-H. Lin, and H.-J. Su, "Lattice coding for the vector fading paper problem," in Proc. IEEE Information Theory Workshop, Tahoe city, CA., USA, Sept. 2-6, 2007.

[17] S.-C. Lin, "Coding for vector dirty-paper channels: Theory, implementation, and duality," Ph.D. dissertation, National Taiwan University, 2007.

[18] J. H. Conway and N. J. A. Sloane, Sphere packings, lattices, and groups. New York: Springer-Verlag, 1988.

[19] A. D. Murugan, H. El-Gamal, M. O. Damen, and G. Caire, "A unified framework for tree search decoding: rediscovering the sequential decoder," IEEE Trans. Inform. Theory, vol. 52, no. 3, pp. 933-953, 2006.

[20] K. R. Kumar and G. Caire, "Construction of Structured LaST Codes," in IEEE International Symposium on Information Theory (ISIT), 2006, pp. 2834-2838.

[21] O. Lieberman, "A Laplace approximation for the distribution of a ratio of quadratic forms in normal variables," J. Amer. Statist. Assoc, vol. 89, pp. 924-928, 1994.

[22] P. Billingsley, Probability and measure, 3rd ed. J. Wiley and Sons, 1995.

[23] M. Kendall and A. Stuart, The advanced theory of statistics. London : C. Griffin, 1977. 


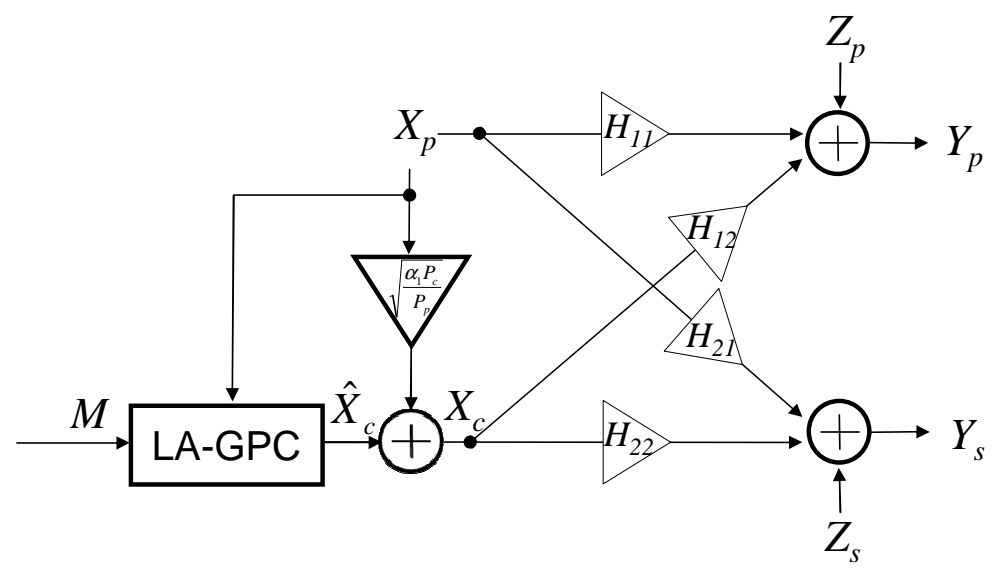

Fig. 1. The model of the cognitive radio channel.

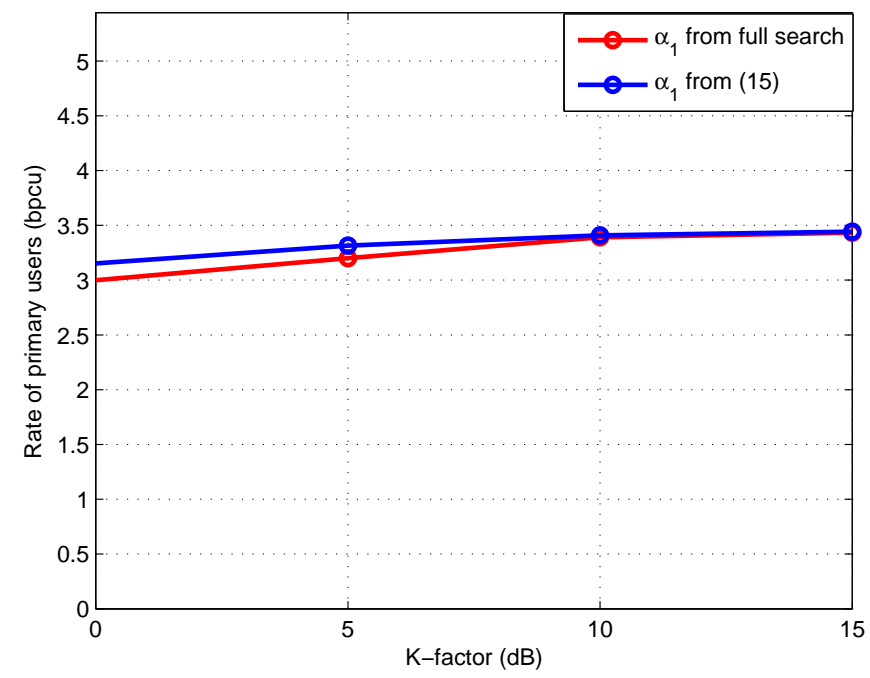

Fig. 2. Comparison of the primary user's ergodic capacities under full search and the proposed method with $P_{c}=P_{p}=10$ and $\sigma_{Z_{s}}^{2}=\sigma_{Z_{p}}^{2}=1$. 


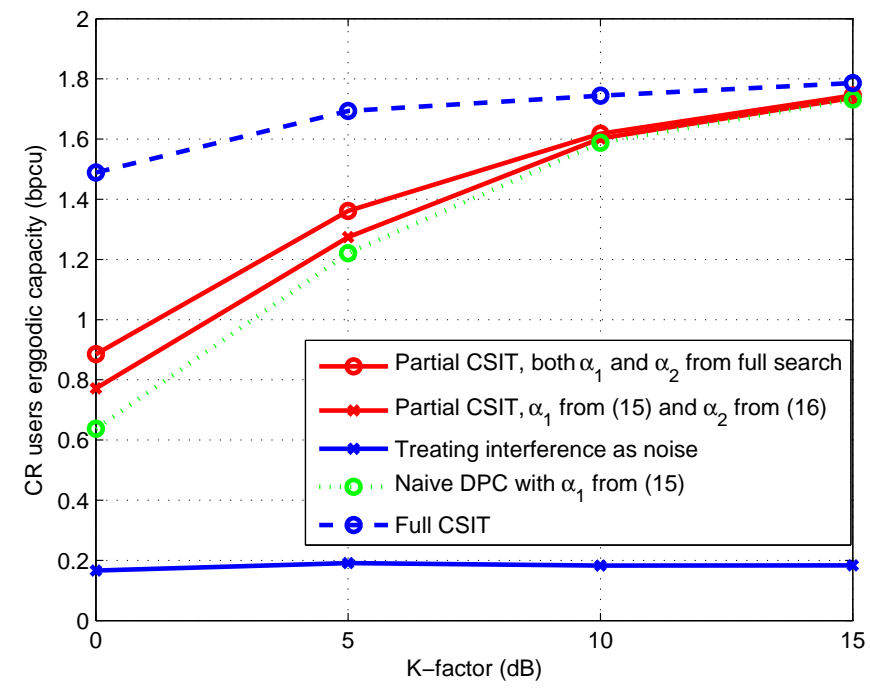

Fig. 3. Comparison of the CR user's ergodic capacities under full search and the proposed method with $P_{c}=P_{p}=10$ and $\sigma_{Z_{s}}^{2}=\sigma_{Z_{p}}^{2}=1$.

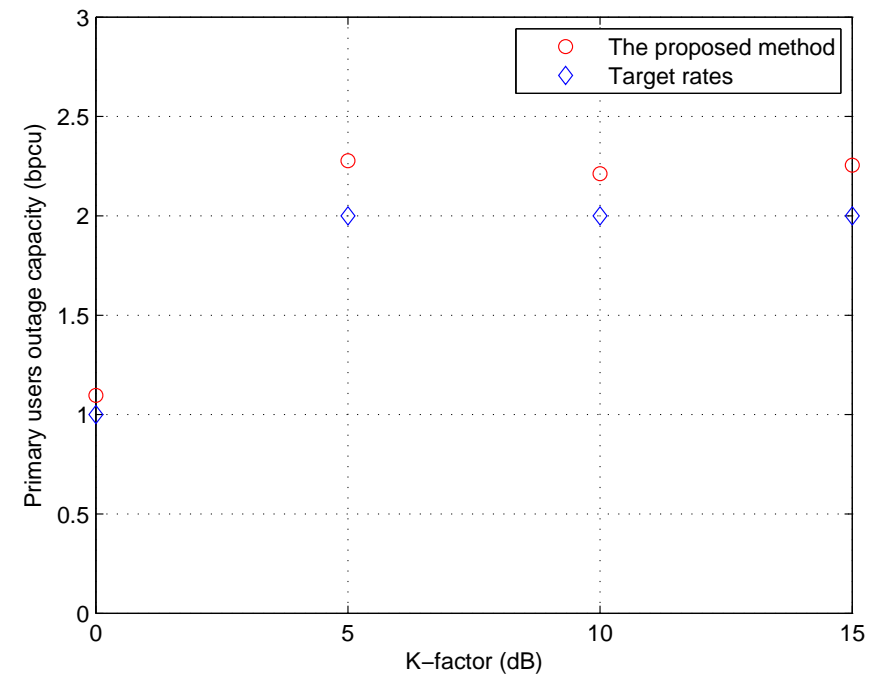

Fig. 4. Comparison of the primary user's outage capacities obtained by the proposed method and the target rates with $P_{c}=P_{p}=10$ and $\sigma_{Z_{s}}^{2}=\sigma_{Z_{p}}^{2}=1$. 


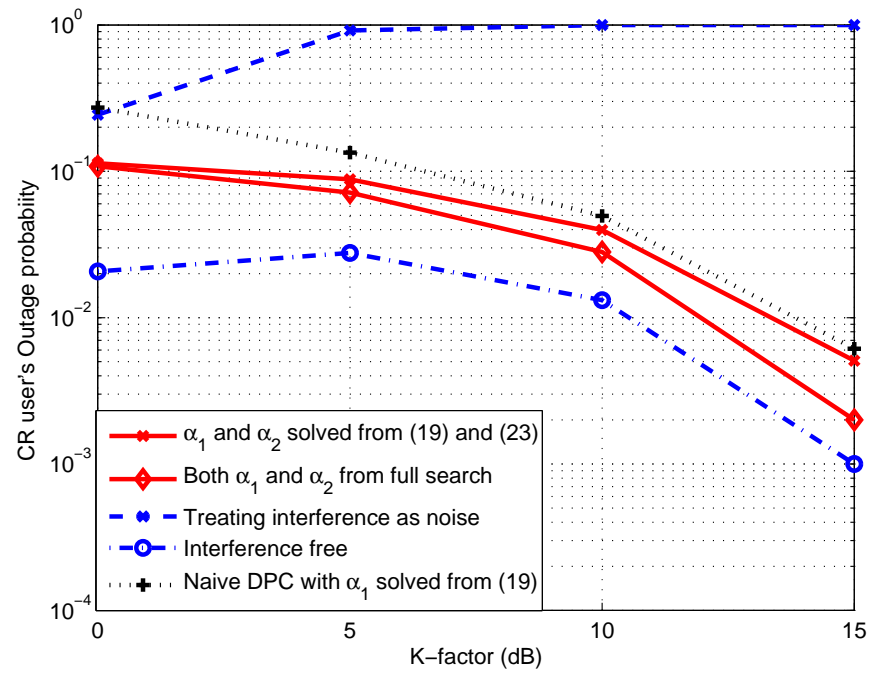

Fig. 5. Comparison of the outage probabilities with $\alpha_{2}$ from full search and the approximation with $P_{c}=P_{p}=10$ and $\sigma_{Z_{s}}^{2}=\sigma_{Z_{p}}^{2}=1$.

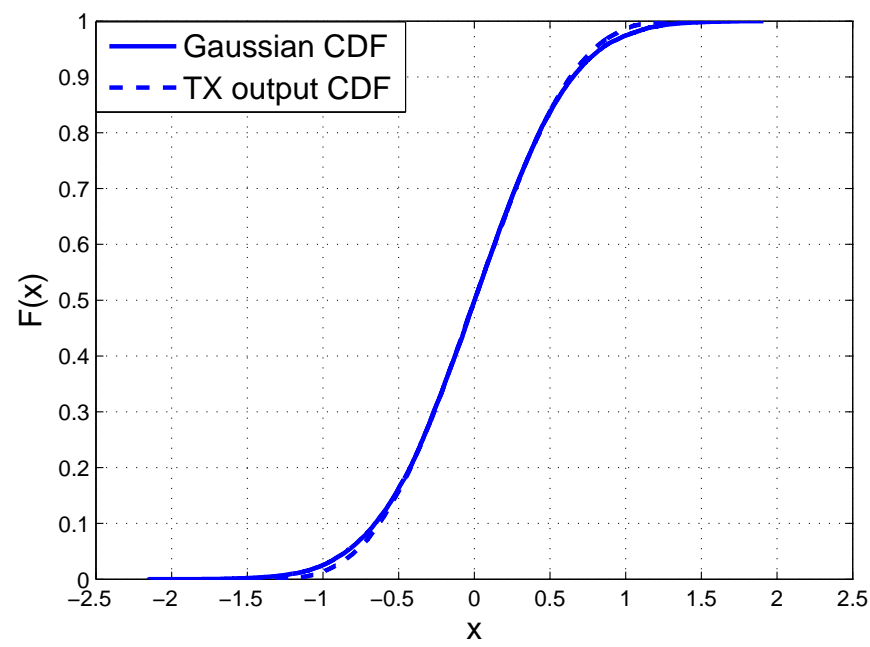

Fig. 6. The Gaussianity of the CR transmitted signal using the nested lattice code. 


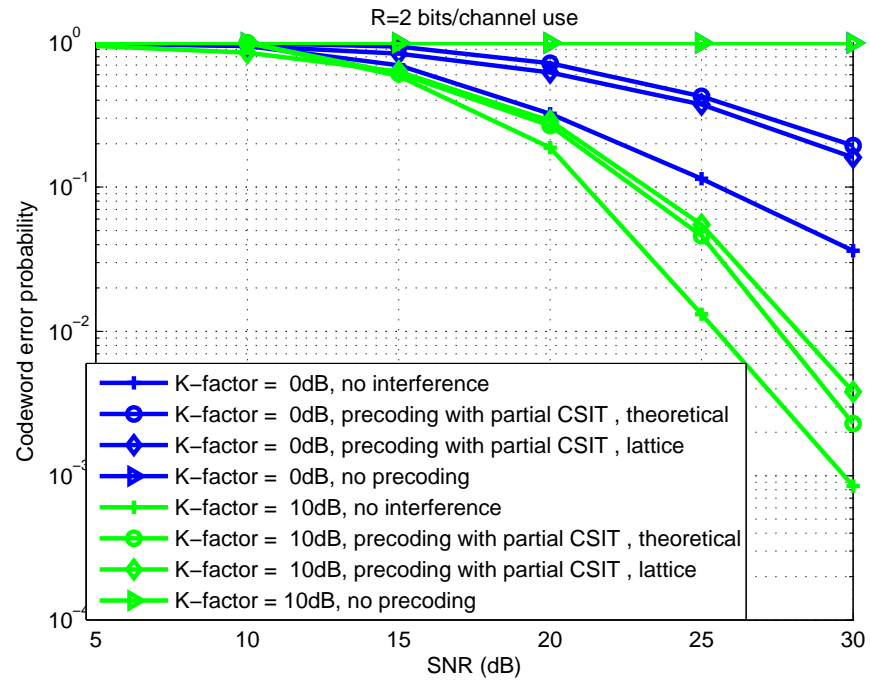

Fig. 7. Comparison of the outage probabilities with rate $=2 \mathrm{bpcu}$.

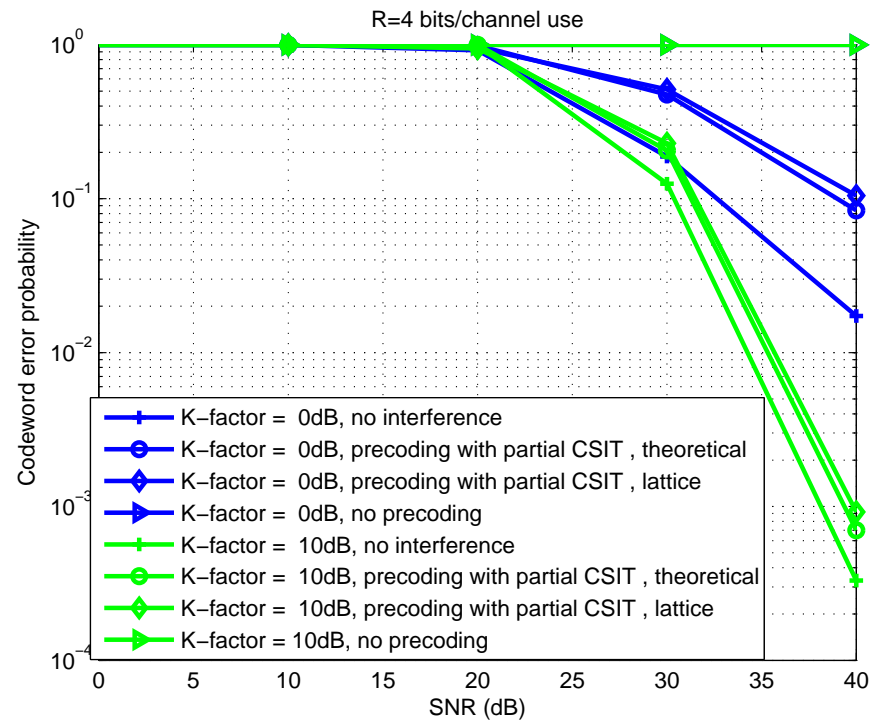

Fig. 8. Comparison of the outage probabilities with rate $=4 \mathrm{bpcu}$. 